\title{
EDITORIAL
}

\section{Cardiac function and obesity}

R S Vasan

Heart 2003;89:1 127-1129

Obesity is increasing around the world, and with it the risk of cardiovascular disease

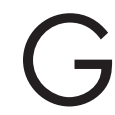
iven the rising global epidemic of obesity, it is likely that adverse health consequences of excess adiposity will escalate in the future. In this context, several investigators have described the adverse effects of obesity on the heart. ${ }^{2}$ Obesity has been linked to a spectrum of cardiovascular changes ranging from a hyperdynamic circulation, ${ }^{2}$ through subclinical cardiac structural changes $^{4}$ to overt heart failure. ${ }^{5}$

\section{OBESITY AND ALTERED CARDIAC LOADING CONDITIONS}

Obesity is associated with haemodynamic overload. $^{23}$ The increased metabolic demand imposed by the expanded adipose tissue and augmented fat-free mass in obesity results in a hyperdynamic circulation with increased blood volume. In addition to the increased preload, left ventricular (LV) afterload is also elevated in obese individuals due to both increased peripheral resistance and greater conduit artery stiffness. Right ventricular afterload may be increased, presumably due to associated sleep disordered breathing and LV changes. ${ }^{3}$

\section{OBESITY AND CARDIAC REMODELLING}

Obesity is associated with atrial and ventricular remodelling (fig 1), known precursors of atrial and ventricular dysfunction, respectively. Obese individuals have larger left atrial size, ${ }^{7}$ related both to an expanded intravascular volume and to altered LV filling properties (see below). Both eccentric and concentric patterns of LV hypertrophy $(\mathrm{LVH})$ have been described in overweight people ${ }^{48}$ with the former being more common. ${ }^{8}$ The extent of cardiac remodelling in obesity increases with its severity and duration, ${ }^{3}$ and is exacerbated by concomitant hypertension. ${ }^{4}$

\section{CARDIAC SYSTOLIC AND DIASTOLIC FUNCTION IN OBESITY}

Cardiac output is often higher in obesity, due to an augmented stroke volume and an increase in heart rate. ${ }^{2}$ Ventricular systolic function as assessed by ejection fraction or with load independent measures such as mid wall fractional shortening is usually normal in obesity. ${ }^{9}$ The increased resting LV end diastolic volume in obesity has been interpreted as evidence of enhanced recruitment of preload reserve (Frank-Starling mechanism). Obese people, however, may fail to increase their ejection fraction with exercise.

Obesity adversely impacts cardiac diastolic function. In a recent large community based investigation, body mass index (BMI) emerged as a key correlate of impaired diastolic filling indexes. ${ }^{10}$ Obesity can alter LV filling indexes because of altered loading conditions (see above), as well as due to an increased LV mass that can adversely impact the passive filling properties of the ventricle. ${ }^{3}$

In this context, the study by Pascual and colleagues in this issue of the Heart assumes significance. ${ }^{11}$ The investigators observed an increased mitral valve pressure half-time and decreased deceleration slope of the early transmitral filling wave in obese women. They interpret these observations as indicating subclinical diastolic dysfunction representing impaired LV relaxation. They also note an increase in ejection phase indices, consistent with some prior reports.

An important strength of the investigation by Pascual and colleagues ${ }^{11}$ is the selection of obese women without co-morbid conditions such as hypertension, diabetes or dyslipidaemia, a design that eliminates confounding by sex and these disease conditions. However, important methodological and conceptual limitations of their approach should be noted. The sample is presumably hospital based, small sized, and exclusively female. Hence, the findings may not be generalisable to the average obese person in the community in whom risk factors frequently cluster. The authors do not provide data on the duration of obesity and its pattern, the presence of impaired glucose tolerance, and LV mass (all factors that can influence diastolic function), or information about the reproducibility of the echocardiographic measurements. Furthermore, the investigators compared 21 different echocardiographic measurements across the four BMI categories, raising the issue of multiple statistical testing.

A more serious limitation is the interpretation that changes in LV diastolic "filling" indexes constitute alterations in myocardial diastolic "function". Transmitral Doppler flow patterns are dependent on multiple interrelated factors, including the rate and extent of ventricular relaxation, ventricular suction, net atrioventricular compliance, mitral valve inertance, atrial systolic function, and left atrial pressure. ${ }^{12}$ Thus, the observation of an increased mitral pressure half-time with increasing BMI in the present report may be explained partly by the increase in LV end diastolic volume in obese individuals, ${ }^{13}$ and need not indicate any change in intrinsic myocardial relaxation. Additionally, as noted by the authors, prior cross sectional studies of obese individuals have reported increased, decreased or unchanged transmitral E wave velocities, with variable effects on the derived E/A ratios. Such disparities in results can arise because transmitral flow indexes are notoriously sensitive to loading conditions, and may change over time according to the degree and stage of diastolic dysfunction. ${ }^{12}$ 


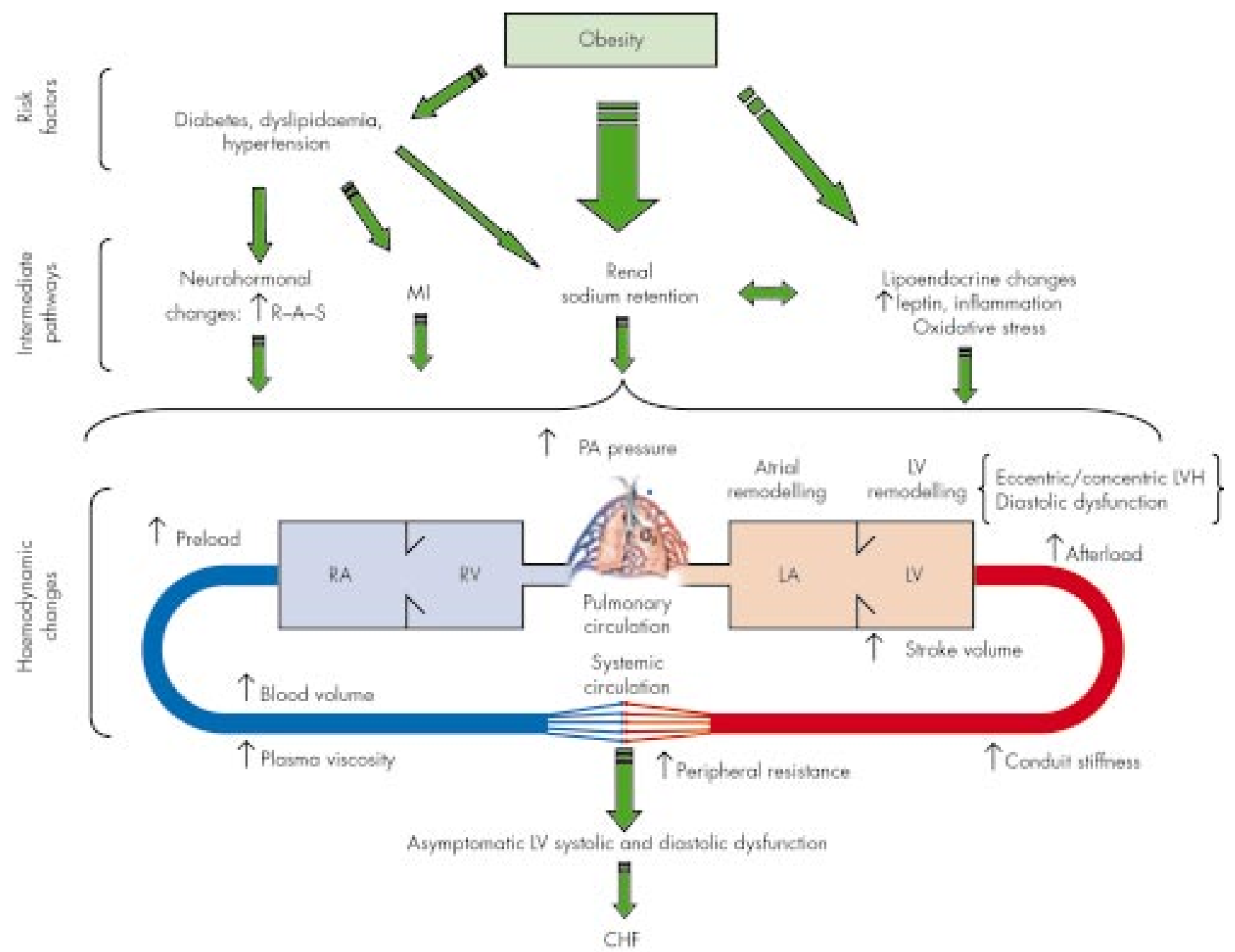

Figure 1 Obesity and cardiac dysfunction: mechanisms. CHF, congestive heart failure; LA and LV, left atrium and ventricle, respectively; LVH, left ventricular hypertrophy; MI, myocardial infarction; PA, pulmonary artery; RA and RV, right atrium and ventricle, respectively; R-A-S, renin-angiotensin system.

To address some of these challenges in the assessment of ventricular diastolic function, investigators have used elaborate research protocols that combine measurement of transmitral flow patterns with evaluations of pulmonary venous flows, mitral annular motion on tissue Doppler imaging and/or the velocity of transmitral flow propagation with colour $\mathrm{M}$ mode echocardiography. ${ }^{12}$ Pascual and colleagues ${ }^{11}$ did not perform such a detailed characterisation of ventricular diastolic filling. So while it can be inferred from their study that diastolic filling patterns are modified in obesity, it is unclear if ventricular diastolic function per se is altered. Future investigations that perform more comprehensive non-invasive evaluation of LV diastolic filling along with assessment of long axis ventricular function, and that include an exercise component, are warranted to understand better the impact of obesity on cardiac function.

\section{MECHANISMS UNDERLYING THE CARDIOMYOPATHY OF OBESITY}

The caveats noted above should not be interpreted to indicate that obesity does not influence LV diastolic function. Indeed, a substantial body of evidence supports such a premise. Obesity can result in LV diastolic dysfunction by promoting other risk factors such as hypertension, dyslipidemia, and diabetes mellitus. Obesity is associated with neuroendocrine activation, renal sodium retention, and heightened systemic oxidative stress. ${ }^{14}$ Adipose tissue is also a major source of proinflamma- tory cytokines. ${ }^{15}$ All these mechanisms can contribute to pathogenesis of LV dysfunction in obesity.

Recent experimental investigations have unravelled the molecular mechanisms that may underlie the cardiomyopathy of obesity. A direct lipotoxic effect on the heart has been suggested with the demonstration of cardiac steatosis and lipoapoptosis in animal models. ${ }^{16}{ }^{17}$ A recent analysis of the cardiac transcriptome in an animal model of obesity suggests profound activation of specific genes in the ventricular and atrial myocardium (for example, MMP-9) very early in response to overfeeding. ${ }^{18}$ The relevance of these exciting findings to humans merits further exploration.

\section{PREVENTIVE IMPLICATIONS}

Recent investigations have led to a paradigm shift in our understanding of the cardiomyopathy of obesity. While initial investigations focused on cardiac dysfunction in the setting of morbid obesity $\left(\mathrm{BMI} \geqslant 40 \mathrm{~kg} / \mathrm{m}^{2}\right),{ }^{3}$ contemporary studies have demonstrated a more continuous gradient of risk of heart failure associated with excess weight, ${ }^{5}$ and have emphasised the occurrence of ventricular remodelling in overweight children. ${ }^{19}$ Thus, promotion of optimal body weight and blood pressure in children and adults through lifestyle measures such as adequate exercise and proper nutrition may be the best measure to prevent the cardiomyopathy of obesity.

\section{ACKNOWLEDGEMENTS}

This work was supported by NIH/NHLBI NOl-HC-25195 and K24-HL-04334. 


\section{REFERENCES}

1 World Health Organization. Obesity: preventing and managing the global epidemic: report of a WHO Consultation on Obesity, Geneva 3-5 June, 1997. Geneva: World Health Organization, 1998.

2 Alexander JK. Obesity and the heart. Heart Dis Stroke 1993;2:317-21.

3 Alpert MA. Obesity cardiomyopathy: pathophysiology and evolution of the clinical syndrome. Am J Med Sci 2001;321:225-36.

4 Laver MS, Anderson KM, Levy D. Separate and joint influences of obesity and mild hypertension on left ventricular mass and geometry: the Framingham heart study. J Am Coll Cardiol 1992;19:130-4.

5 Kenchaiah S, Evans JC, Levy D, et al. Obesity and the risk of heart failure. N Engl J Med 2002;347:305-13

6 Sutton-Tyrrell K, Newman A, Simonsick EM, et al. Aortic stiffness is associated with visceral adiposity in older adults enrolled in the study of health, aging, and body composition. Hypertension 2001;38:429-33.

7 Gottdiener MD, Reda MS, Williams MS, et al. Left atrial size in hypertensive men: influence of obesity, race and age. J Am Coll Cardiol 1997:29:651-8.

8 de Simone G, Devereux RB, Roman Mv, et al. Relation of obesity and gender to left ventricular hypertrophy in normotensive and hypertensive adults. Hypertension 1994;23:600-6.

9 de Simone G, Devereux RB, Mureddu GF, et al. Influence of obesity on left ventricular midwall mechanics in arterial hypertension. Hypertension 1996;28:276-83.
10 Fischer $\mathbf{M}$, Baessler A, Hense HW, et al. Prevalence of left ventricular diastolic dysfunction in the community: results from a Doppler echocardiographic-based survey of a population sample. Eur Heart $J$ 2003;24:320-8.

11 Pascual M, Pascual DA, Soria F, et al. Effects of isolated obesity on systolic and diastolic left ventricular function. Heart 2003:89: 1 152-6.

12 Garcia MJ, Thomas JD, Klein AL. New Doppler echocardiographic applications for the study of diastolic function. J Am Coll Cardiol 1998;32:865-75.

13 Alpert MA, Lambert CR, Terry BE, et al. Influence of left ventricular mass on left ventricular diastolic filling in normotensive morbid obesity. Am Heart J 1995;130:1068-73.

14 Keaney JF, Jr, Larson MG, Vasan RS, et al. Obesity and systemic oxidative stress: clinical correlates of oxidative stress in the Framingham study. Arterioscler Thromb Vasc Biol 2003;23:434-9.

15 Lyon CJ, Law RE, Hsueh WA. Minireview: adiposity, inflammation, and atherogenesis. Endocrinology 2003;144:2195-200.

16 Zhou YT, Grayburn P, Karim A, et al. Lipotoxic heart disease in obese rats: implications for human obesity. Proc Natl Acad Sci U S A rats: impo: 97: 1784-9.

17 Chiu HC, Kovacs A, Ford DA, et al. A novel mouse model of lipotoxic cardiomyopathy. J Ćlin Invest 2001;107:813-22.

18 Philip-Couderc P, Smih F, Pelat $M$, et al. Cardiac transcriptome analysis in obesity-related hypertension. Hypertension 2003;41:414-21.

19 Gutin B, Treiber F, Owens S, et al. Relations of body composition to left ventricular geometry and function in children. J Pediatr ventricular geometry

\section{IMAGES IN CARDIOLOGY}

Right atrium and testis tumour: is there a link?

A 26 year old man with a recently diagnosed germinal testicular tumour treated by orchidectomy and chemotherapy started to complain of chest pain and fever after insertion of a port-a-cath. Because blood culture revealed methicillin resistant Staphylococcus aureus (MRSA), the port-a-cath was removed. Transoesophageal echocardiography (panel A) showed a mass in the right atrium. At this time, chemotherapy was stopped and antibiotics were started.

Four possible diagnoses were proposed: cardiac metastasis of the testis neoplasm, early heart tumour, or endocarditis or thrombus related to the porta-cath insertion.

Cine magnetic resonance imaging showed the presence of a single very mobile mass with a large base implanted on the posterior atrial wall (panel B). There was no extension to the vena cava. On Tl imaging, the mass appeared homogenous (panel C). After injection of $15 \mathrm{ml}$ Gd-DTPA, there was no enhancement of the mass, suggesting a benign nature (panel D).

Lateral thoracotomy and atriotomy without extracorporeal circulation was performed to extract the mass. Pathology confirmed the thrombotic nature of the tumour. This very large thrombus was probably related to the traumatic insertion of the malpositioned port-a-cath. Following surgery, antibiotics and anticoagulants were continued, with a favourable clinical outcome for the patient.

J Kefer

F Dumont

B Gerber

joelle.kefer@clin.ucl.ac.be
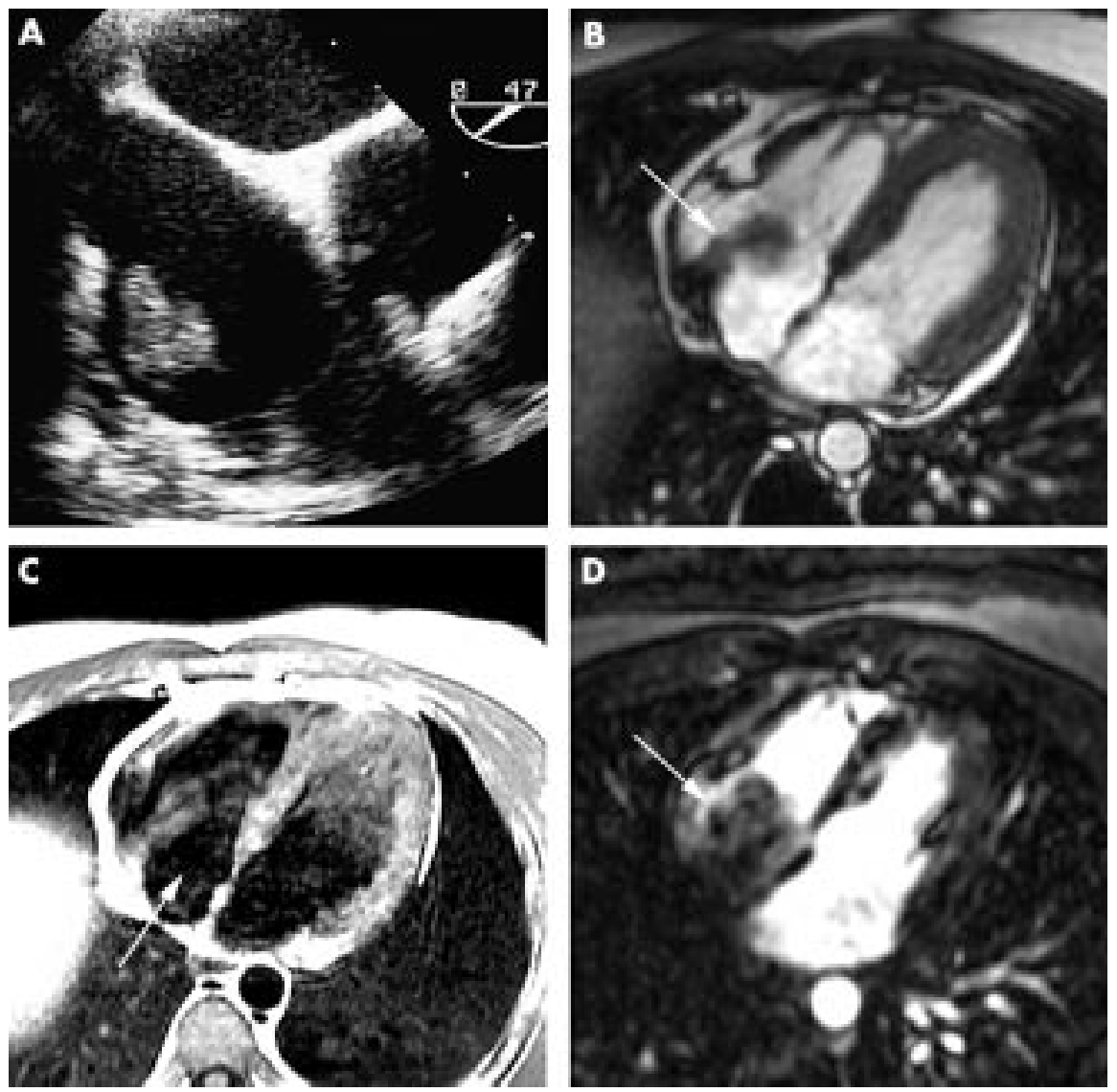